\title{
Morphometric Evaluation of the Middle Turbinate in Relation to Endoscopic Sinus Surgery
}

\author{
Tulika Gupta, Anjali Aggarwal, Daisy Sahni
}

\begin{abstract}
Objective: Partial middle turbinectomy during endoscopic sinus surgery is done for better access and to avoid postoperative complications of lateralization of the middle turbinate. There are only few cadaveric studies available in literature describing the middle turbinate morphometry with surgical perspective. Therefore, the present study was planned to record morphometry and relevant surgical distances for the middle turbinate.
\end{abstract}

Materials and methods: Thirty-two midsagittal sections of cadaver heads from adult males were dissected. Middle turbinate measurements and shapes were taken. Reference distances and angles were measured.

Results: The average length of the middle turbinate was 20.4 $\pm 3.9 \mathrm{~mm}$, while the height of the middle turbinate was taken at three specific points $(13.1,9.7,6.1 \mathrm{~mm})$. The most common shape found was the type 2 . The mean value for the distance between limen nasi and the anterior and posterior attachments of the middle turbinate was found to be $32.1 \pm 3.2 \mathrm{~mm}$, with mean angle of $65^{\circ}$ with the horizontal and $43.6 \pm 4.2 \mathrm{~mm}$, with mean angle of $39.4^{\circ}$ with the horizontal respectively.

Conclusion: This baseline data may aid endoscopic surgeons in better planning and execution of the middle turbinate resection.

Keywords: Middle turbinate resection, Endoscopic sinus surgery, Limen nasi.

How to cite this article: Gupta T, Aggarwal A, Sahni D. Morphometric Evaluation of the Middle Turbinate in Relation to Endoscopic Sinus Surgery. Clin Rhinol An Int J 2012;5(3): 103-106.

Source of support: Nil

Conflict of interest: None declared

\section{INTRODUCTION}

The anatomy of paranasal region and its variations have gained importance because of widespread practice of endoscopic sinus surgery (ESS). Significant advances have been made in endoscopic techniques; however frequent, miscellaneous anatomic variations in the paranasal region increase the risk for possible complications of ESS. ${ }^{1}$ The middle turbinate (MT) is important for optimum physiologic functioning of the nasal cavity and is considered a useful landmark for guidance during ESS. Though it is desirable to preserve the MT during ESS, there are many a situation in which partial middle turbinectomy is warranted either to enhance surgical access to the middle meatus or for postoperative debridement. Most of the previous studies have reported MT variations like concha bullosa, accessory concha, etc. ${ }^{2-5}$ but detail morphometry of the MT have been described by very few authors. ${ }^{5,6}$ Moreover, most of the available studies on sinonasal anatomy are radiological, ${ }^{2,4,7,8}$ with very few cadaver-based studies. ${ }^{9}$ Therefore, the present study was planned on midsagittal section of cadaveric heads with the aim to collect data related to shape, anatomical measurements and angles related to the MT. This anatomical knowledge can be useful for the surgeons performing ESS and middle turbinectomy.

\section{MATERIALS AND METHODS}

Thirty-two midsagittal sections of cadaver heads from adult males were used for dissection and measurement. The study was approved by institute ethical committee. Any specimen with known nasal pathology was not included in the study. The nasal septum was removed from each of the specimen. The three turbinates were identified. The following measurements were done with the help of digital vernier caliper (accurate up to $0.02 \mathrm{~mm}$ ) and protractor.

A. Dimensions of the MT (Fig. 1)

1. Length of the MT: It was measured from the anterior attachment of the MT to the anterior end of the superior turbinate (posterior attachment of the MT).

2. Height of the MT: It was measured at three points.

a. H1: The height of the MT taken at the anteriormost point of the superior turbinate.

b. H2: The height of the MT taken at the middle portion of the MT.

c. H3: The height of the MT taken at the posterior portion of the MT.

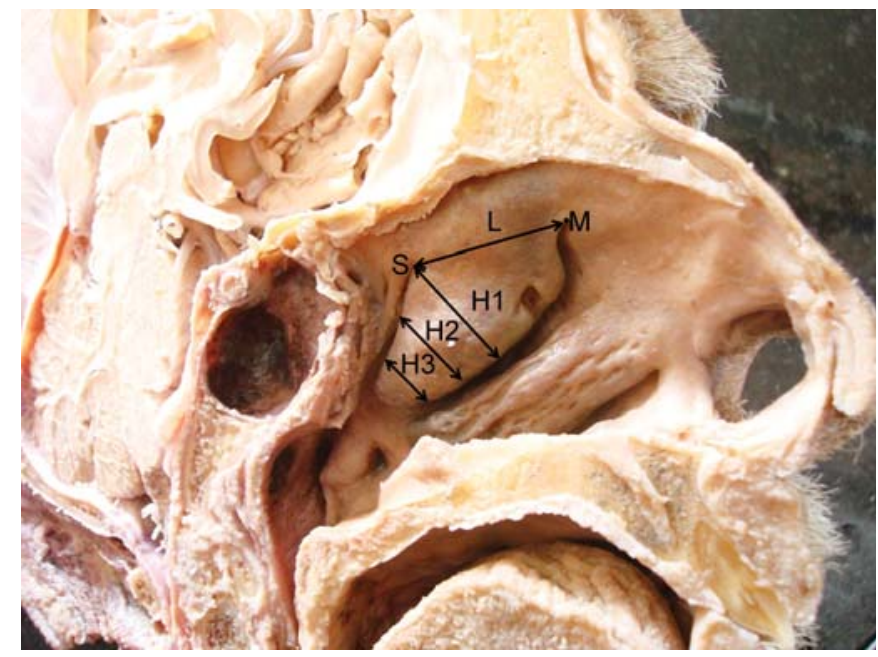

Fig. 1: Various dimensions of the MT 


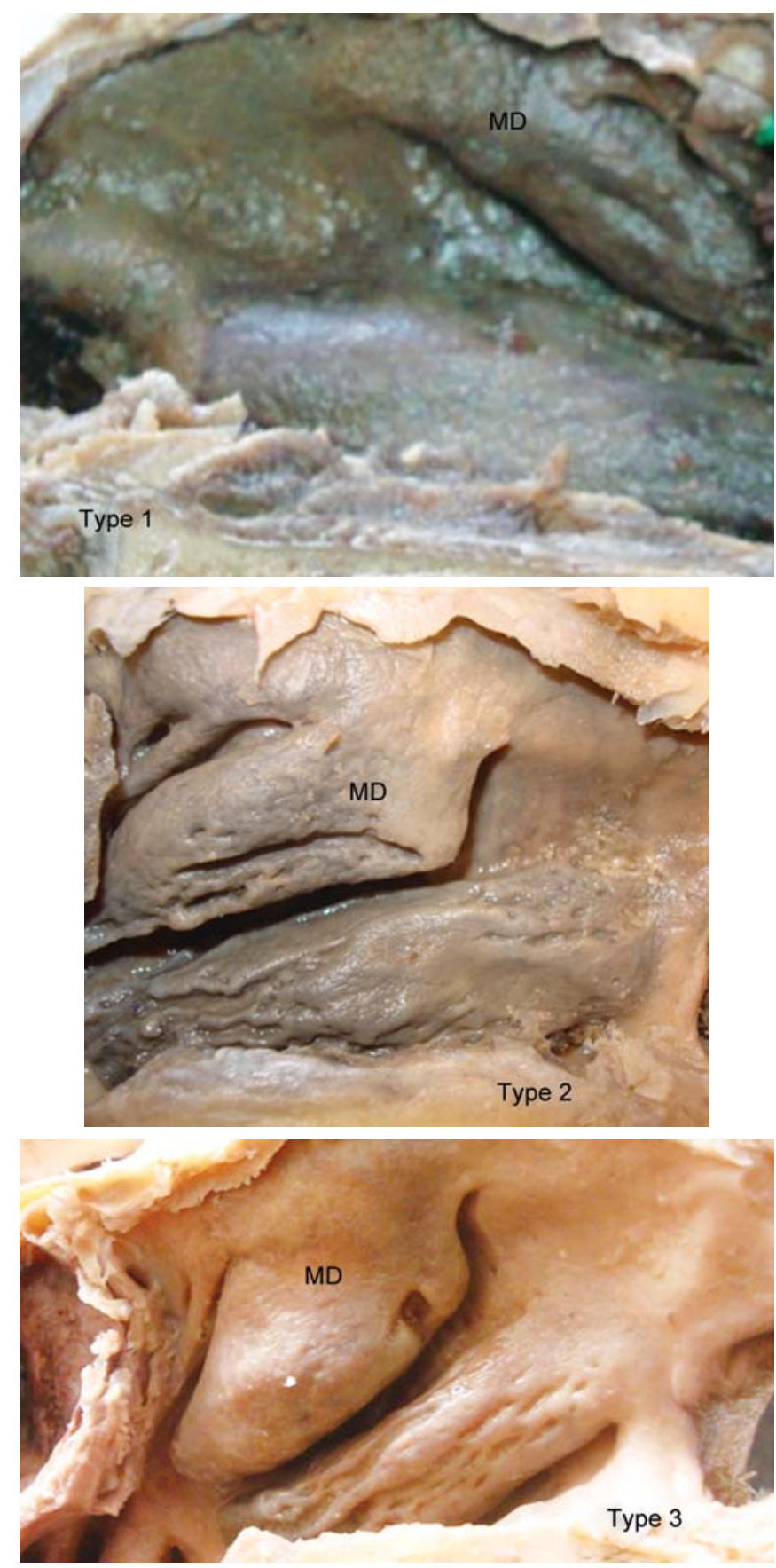

Fig. 2: The three different types of the MT shapes

B. Shape of the MT (Fig. 2): We have followed the classification extended by Lee et al: ${ }^{9}$

1. Type 1: The anterior border of the MT ran directly posteroinferiorly from its attachment to the conchal plate.

2. Type 2: The anterior border of the MT initially coursed inferiorly from the conchal plate then turned in a posteroinferior direction.

3. Type 3: The anterior border of the MT bulged anteriorly before coursing posteroinferiorly.

C. The distances and angles between the limen nasi, the MT and the superior turbinate (Fig. 3)
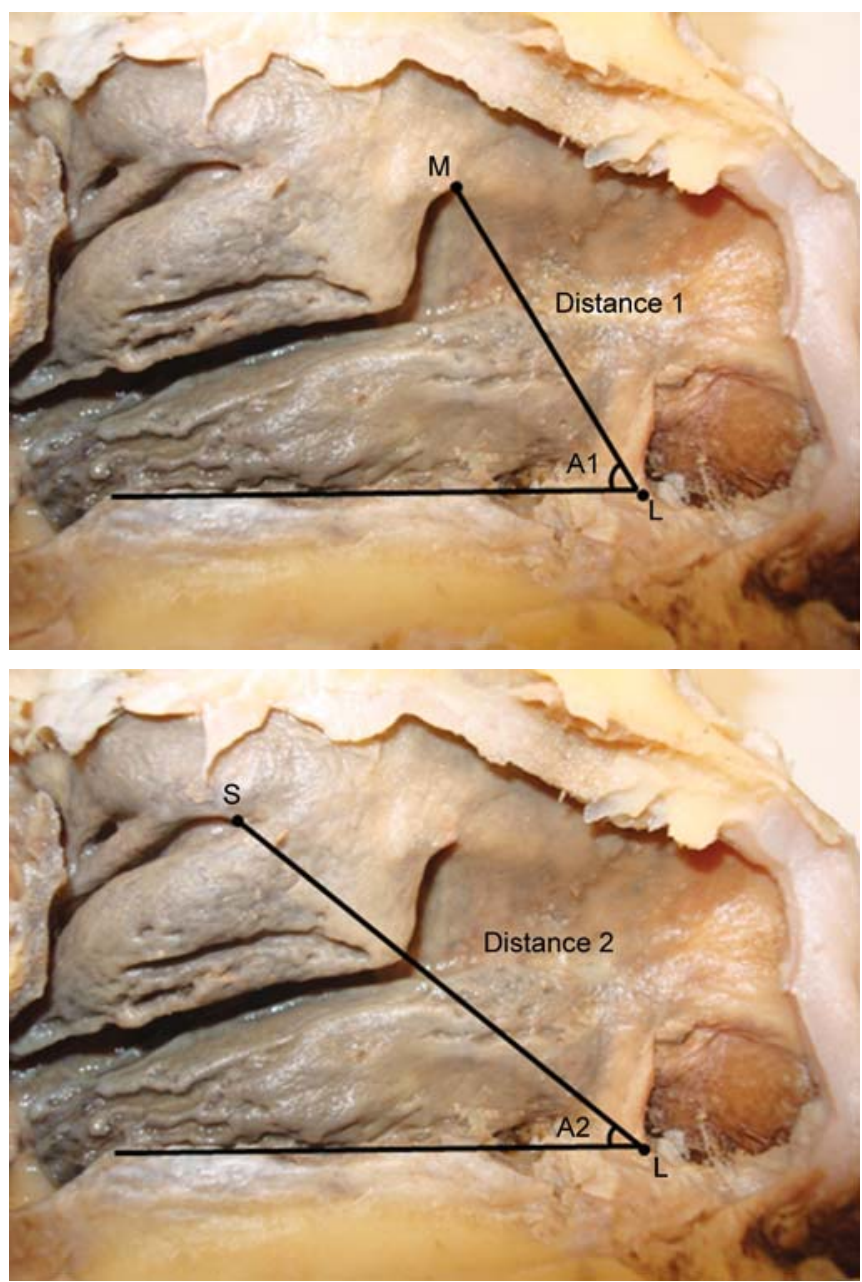

Fig. 3: The distances and angles between the limen nasi, the MT and the superior turbinate

1. Distance 1: The distance between the lower most point of the limen nasi $(\mathrm{L})$ and the anteriormost attachment of the MT (M).

2. Angle 1: The angle between the line passing through the point $\mathrm{L}$ and point $\mathrm{M}$ and the horizontal line passing through the limen nasi.

3. Distance 2: The distance between the lower most point of the limen nasi (L) and the anteriormost attachment of the superior turbinate (S).

4. Angle 2: The angle between the line passing through the point $\mathrm{L}$ and point $\mathrm{S}$ and the horizontal line passing through the limen nasi.

\section{RESULTS}

\section{Dimensions of the MT (Table 1)}

The average length of the MT was $20.4 \pm 3.9 \mathrm{~mm}$, with the range of 10.7 to $28.5 \mathrm{~mm}$. In $90 \%$ of the specimens length of the MT varied between 15 and $25 \mathrm{~mm}$. The mean value for the $\mathrm{H} 1$ was found to be $13.1 \pm 3.2 \mathrm{~mm}$ (range, $5.5-19.5 \mathrm{~mm}$ ). In $77 \%$ of the specimens $\mathrm{H} 1$ was between 10 and $15 \mathrm{~mm}$. H2 ranged from 2.3 to $15.9 \mathrm{~mm}$ with the 
mean of $9.7 \pm 2.8 \mathrm{~mm}$. In huge majority (87\%) of the specimens $\mathrm{H} 2$ was between 6 and $12 \mathrm{~mm}$. The mean height at the posterior portion (H3) was found to be $6.1 \pm 1.9 \mathrm{~mm}$ with the range of 1.5 to $9.5 \mathrm{~mm}$.

\section{Shape of the MT}

The most common shape found was the type 2 . It was present in $70 \%$ of the specimens examined. The MT in $23.3 \%$ of the specimens had the type 1 shape while the type 3 shape was found in only $6.7 \%$ of the specimens.

\section{The Distances and Angles between the Limen Nasi, the MT and the Superior Turbinate (Table 1)}

The mean value for the distance between limen nasi and the anterior attachment of the MT was found to be $32.1 \pm$ $3.2 \mathrm{~mm}$ (range, 26.8-38.6 mm). The distance 1 varied between 30 and $35 \mathrm{~mm}$ in $73 \%$ of cases. The corresponding angle with the horizontal (angle 1) had mean value of $65.3^{\circ}$ $\pm 5.5^{\circ}$, with the range of $50^{\circ}$ to $70^{\circ}$. In $90 \%$ of the heads examined the angle 1 was constantly found to be between $60^{\circ}$ and $70^{\circ}$. The mean value for the distance between limen nasi and the superior turbinate (the posterior attachment of the MT) was $43.6 \pm 4.2 \mathrm{~mm}$ (range, 34.3-49.1 mm). In 87\% of the specimens the distance 2 was between 40 and 50 $\mathrm{mm}$. The angle 2 had mean value of $39.4^{\circ} \pm 3.1^{\circ}$ (range, $33^{\circ}-48^{\circ}$ ). In $83 \%$ of the specimens the angle 2 was between $38^{\circ}$ and $42^{\circ}$.

\section{DISCUSSION}

The turbinates are physiologic structures that participate in the nasal cycle and are important for maintenance of a laminar airflow. The MT is the primary site for impaction of inhaled particles and serves to protect the maxillary and ethmoid sinuses from inhaled air and from the resultant drying effects on sinus mucociliary clearance. ${ }^{10}$ Anterior/ superior portion of the MT is considered to be an important surgical landmark. ${ }^{11}$ Diversity of opinions continues to exist among otolaryngologists regarding potential benefits of preservation or resection of normal MT during endoscopic ethmoidectomy. MT resection can cause diminished humidification and filtration of inspired air, hyposmia and loss of important anatomical landmark. Conservative partial middle turbinectomy is a good alternative which preserve a portion of MT as a landmark, however MT remnant could become floppy and lateralize, causing frontal recess obstruction and frontal sinusitis. ${ }^{12}$ Many surgical techniques of safe partial MT resection are described to avoid lateralization of MT remnant. ${ }^{12-14}$ According to the study by Giacchi et al, ${ }^{12}$ anterior/inferior one-third to one-half of the turbinate should be removed while preserving superior and lateral attachments. Other authors ${ }^{13,14}$ prefer to preserve superior half of the MT. In our study the mean length of the MT was measured between the anterior attachments of the middle and superior turbinate, this length attaches the MT to the lateral nasal wall. The length of the MT was found to be about $2 \mathrm{~cm}$, while in $90 \%$ of the specimens it varied from 1.5 to $2.5 \mathrm{~cm}$. Lee et al in their study of 101 hemisected cadaveric heads have reported the similar value $(18.5 \mathrm{~mm})$ for the MT length. The surgeon should consider these values while deciding the resection line because extensive removal of anterior portion of the MT can decrease the stability of the MT remnant. ${ }^{9}$ Moreover, surgeon should be careful as not to remove the portion superior to the horizontal line connecting anterior attachments of the MT and the superior turbinate, where olfactory nerve filaments are distributed, as this could result in postoperative hyposmia. ${ }^{15}$

Baseline data regarding the height of the MT at various points may aid the surgeon in better planning of partial MT resection. We found the height of the MT at the anterior

\begin{tabular}{|c|c|c|c|c|c|c|c|c|}
\hline & \multirow[t]{2}{*}{ Length } & \multicolumn{3}{|c|}{ Height } & \multirow[t]{2}{*}{ Distance 1} & \multirow[t]{2}{*}{ Angle 1} & \multirow[t]{2}{*}{ Distance 2} & \multirow[t]{2}{*}{ Angle 2} \\
\hline & & $H 1$ & $\mathrm{H} 2$ & $H 3$ & & & & \\
\hline Minimum & 10.7 & 5.5 & 2.3 & 1.5 & 26.8 & $50^{\circ}$ & 34.3 & $33^{\circ}$ \\
\hline Maximum & 28.5 & 19.5 & 15.9 & 9.5 & 38.6 & $70^{\circ}$ & 49.1 & $48^{\circ}$ \\
\hline Mean & 20.4 & 13.1 & 9.7 & 6.1 & 32.1 & $65.3^{\circ}$ & 43.6 & $39.4^{\circ}$ \\
\hline SD & 3.9 & 3.2 & 2.8 & 1.9 & 3.2 & $5.5^{\circ}$ & 4.2 & $3.1^{\circ}$ \\
\hline
\end{tabular}

All the linear measurements are in millimeter.

- Length of the MT: It was measured from the anterior attachment of the MT to the anterior end of the superior turbinate.

- Height of the MT:

- H1: The height of the MT taken at the anteriormost point of the superior turbinate.

- H2: The height of the MT taken at the middle portion of the MT.

- H3: The height of the MT taken at the posterior portion of the MT.

- Distance 1: The distance between the lower most point of the limen nasi (L) and the anteriormost attachment of the MT (M).

- Angle 1: The angle between the line passing through the points $L$ and $M$ and the horizontal line passing through the limen nasi.

- Distance 2: The distance between the lower most point of the limen nasi (L) and the anteriormost attachment of the superior turbinate (S).

- Angle 2: The angle between the line passing through the point $L$ and point $S$ and the horizontal line passing through the limen nasi.

- SD: Standard deviation. 
end of the superior turbinate to be $13 \mathrm{~mm}$ which is comparable to the value $(10.9 \mathrm{~mm})$ reported by Lee et al. ${ }^{9}$ The mean values for the MT height taken at the middle and the posterior portion (10.6 and $7.7 \mathrm{~mm}$ ) are also similar to those found in the previous study (9.7 and $6.1 \mathrm{~mm}$ ). ${ }^{9}$

Morphologically, MT was classified into three types. In the present study the type 2 was the most common shape with the incidence of $70 \%$. In a study of Koreans cadavers both type 1 (45.3\%) and type 2 (44.2\%) were reported to be equally prevalent in the population. ${ }^{9}$ In type 1 MT the posterior part of the MT is quite large. Therefore, liberal anterior resection can easily destabilize the MT especially when the partial middle turbinectomy and posterior ethmoidectomy is simultaneously performed, which means that the basal lamella is removed. The MT remnant can fall into the nasopharynx as a result of gravity. ${ }^{9}$ Type 3 morphology was present in only $6.7 \%$ of the specimens in the present study. These turbinates appear large and bulging anteriorly on endoscopy. Therefore, an anterior partial middle turbinectomy may be usually required to ensure wider view during surgery and to maintain the patency of the middle meatus postoperatively. ${ }^{9}$

The reference distances and angles were measured from the lower end of the limen nasi as this is the anteriormost landmark and an easily identifiable reference point while inserting the endoscope or other surgical instruments in ESS. The mean distance from limen nasi to the anterior attachment of the MT was about $32 \mathrm{~mm}$ with an angle of $65^{\circ}$ to the horizontal. Other authors have reported this distance as varying from 38 to $50 \mathrm{~mm}^{9,16,17}$ with the mean value for the corresponding angle from $56^{\circ}$ to $68^{\circ}{ }^{9,18}$ The distance 2 from the limen nasi to the anterior attachment of the superior turbinate was found to be about $44 \mathrm{~mm}$ with an angle of about $40^{\circ}$ with the horizontal. The values are comparable to those $\left(49 \mathrm{~mm}, 45^{\circ}\right)$ reported in previous study.

The MT is a key landmark in the nasal cavity. Therefore, baseline data regarding the shapes and dimensions of MT as well as reference distance and angles will be useful in accurate diagnostic and surgical management of the patients undergoing ESS.

\section{REFERENCES}

1. Cukurova I, Yaz A, Gumussoy M, et al. A patient presenting with concha bullosa in another concha bullosa: A case report. J Med Case Rep 2012 Mar 26;6:87.

2. Aksungur EH, Biçakçi K, Inal M, et al. CT demonstration of accessory nasal turbinates: Secondary middle turbinate and bifid inferior turbinate. Eur J Radiol 1999 Sep;31(3):174-76.
3. Joe JK, Ho SY, Yanagisawa E. Documentation of variations in sinonasal anatomy by intraoperative nasal endoscopy. Laryngoscope 2000 Feb;110(2 Pt 1):229-35.

4. Ozcan KM, Selcuk A, Ozcan I, et al. Anatomical variations of nasal turbinates. J Craniofac Surg 2008 Nov;19(6):1678-82.

5. Aydil U, Ozçelik T. Unilateral agenesis of middle nasal turbinate. J Laryngol Otol 2010 Apr; 124(4):447-49.

6. Bolger WE, Butzin CA, Parsons DS. Paranasal sinus bony anatomic variations and mucosal abnormalities: CT analysis for endoscopic sinus surgery. Laryngoscope 1991 Jan;101(1 Pt 1):56-64.

7. Stallman JS, Lobo JN, Som PM. The incidence of concha bullosa and its relationship to nasal septal deviation and paranasal sinus disease. Am J Neuroradiol 2004 Oct;25(9):1613-18.

8. Badia L, Lund VJ, Wei W, et al. Ethnic variation in sinonasal anatomy on CT scanning. Rhinology 2005 Sep; 43(3):210-14.

9. Lee HY, Kim CH, Kim JY, et al. Surgical anatomy of the middle turbinate. Clin Anat 2006 Sep;19(6):493-96.

10. Kennedy DW. Middle turbinate resection: Evaluating the issues - should we resect normal middle turbinates? Arch Otolaryngol Head Neck Surg 1998 Jan;124(1):107.

11. Jacobs JB. 100 years of frontal sinus surgery. Laryngoscope 1997;107(11 Suppl 83):1-36.

12. Giacchi RJ, Lebowitz RA, Jacobs JB. Middle turbinate resection: Issues and controversies. Am J Rhinol 2000; 14(3):193-97.

13. Lawson W. The intranasal ethmoidectomy: An experience with 1,077 procedures. Laryngoscope 1991;101:367-71.

14. LaMear WR, Davis WE, Templer JW, et al. Partial endoscopic middle turbinectomy augmenting functional endoscopic sinus surgery. Otolaryngol Head Neck Surg 1992;107:382-89.

15. Kim KS, Choi YS, Kim HJ, et al. The risk of olfactory disturbance from conchal plate injury during ethmoidectomy. Am J Rhinol 2003;17(5):307-10.

16. Lang J. Clinical anatomy of the nose, nasal cavity and paranasal sinuses. New York: Thieme Medical 1989:117-30.

17. Turgut S, Gumusalan Y, Arifoglu Y, et al. Endoscopic anatomic distances on the lateral nasal wall. J Otolaryngol 1996 Dec;25(6):371-74.

18. Unlü HH, Tekdemir I, Ersoy M, et al. Morphometric evaluation of paranasal sinuses for endoscopic sinus surgery. Ann Anat 1994 Apr;176(2):193-99.

\section{ABOUT THE AUTHORS}

\section{Tulika Gupta (Corresponding Author)}

Assistant Professor, Department of Anatomy, Postgraduate Institute of Medical Education and Research, Chandigarh, India, Phone: +91-2755203, e-mail: tulikag11@yahoo.com

\section{Anjali Aggarwal}

Assistant Professor, Department of Anatomy, Postgraduate Institute of Medical Education and Research, Chandigarh, India

\section{Daisy Sahni}

Professor, Department of Anatomy, Postgraduate Institute of Medical Education and Research, Chandigarh, India 